Tropical Journal of Pharmaceutical Research February 2016; 15 (2): 327-333

ISSN: $1596-5996$ (print); 1596-9827 (electronic) (c) Pharmacotherapy Group, Faculty of Pharmacy, University of Benin, Benin City, 300001 Nigeria.

All rights reserved.

\title{
Ameliorative Effect and Underlying Mechanisms of Total Triterpenoids from Psidium guajava Linn (Myrtaceae) Leaf on High-Fat Streptozotocin-induced Diabetic Peripheral Neuropathy in Rats
}

\author{
Xiaokang Wang ${ }^{1}$, Kaihe $\mathrm{Ye}^{1}$, Yanqing $\mathrm{Lv}^{1}$, Songcheng Wei ${ }^{1}$, Xiucun $\mathrm{Li}^{1}$, Jinjin $\mathrm{Ma}^{1}$, \\ Xiaoqi Zhang ${ }^{2}$ and Chunling $\mathrm{Ye}^{1 *}$ \\ ${ }^{1}$ Pharmacy College, Jinan University, Guangzhou 510632, ${ }^{2}$ Institute of Traditional Chinese Medicine \& Natural Products and \\ JNU-HKUST Joint Laboratory for Neuroscience \& Innovative Drug Research, Pharmacy College, Jinan University, Guangzhou \\ 510632, China
}

*For correspondence: Email: yechunling2005@163.com; Fax: +86 2085223843

Received: 14 September 2015

Revised accepted: 11 January 2016

\begin{abstract}
Purpose: To investigate whether the total triterpenoids extracted from Psidium Guajava leaves (TTPGL) attenuate the development of diabetic peripheral neuropathy in rats by regulating the NF-KB pathway of the inflammatory process and its signaling mediators.

Methods: All the Sprague Dawley rats used were maintained in a clean environment on a $12 \mathrm{~h}$ light/12 $h$ dark cycle. High-fat feeding and intraperitoneal injection of $40 \mathrm{mg} / \mathrm{kg}$ streptozotocin (STZ) were used to induce diabetes in the rats. The rats were randomly divided into 5 groups: diabetic mellitus (DM) group; TTPGL - 30 group, TTPGL - 60 group and TTPGL - 120 group treated by intragastric administration (i.g) with 30, 100 and $120 \mathrm{mg} / \mathrm{kg} /$ day TTPGL, respectively. The well-established drug, rosiglitazone (RSG, $3 \mathrm{mg} / \mathrm{k} / \mathrm{d}$, i.g.), was used as positive control. Normal rats served as control group. Nerve conduction velocity and sensitive tests were measured on weeks 1, 4 and 8 . After 8 weeks administration, expression of pro-inflammatory molecules (TNF - $\alpha, I L-6$ and iNOS) and tissue proteins (Akt, IKKa, and NF - KB - p65) were evaluated to assess biochemical changes.

Results: Compared to DM group, TTPGL (especially $120 \mathrm{mg} / \mathrm{kg}$ dose) treatment improved $(p<0.05)$ physical functions and provided neuronal protection in high - fat/streptozotocin - induced peripheral neuropathy rats. We found that the expressions of several pro - inflammatory factors such as tumor necrosis factor - $\alpha$ (TNF - $\alpha$ ), IL - 6 and inducible nitric oxide synthase (iNOS) were highly suppressed ( $p$ $<0.05$ or $p<0.01$ ) by TTPGL in sciatic nerve. Mechanism analysis indicated that the ameliorative effect of TTPGL, in part, is through suppression of the expression of pro - inflammatory cytokines by NF - $K B$ pathway mediation.

Conclusion: TTPGL offers a potential therapeutic approach for the treatment of diabetic peripheral neuropathy.
\end{abstract}

Keywords: Triterpenoids, Psidium Guajava, Diabetic peripheral neuropathy, Pro -inflammatory cytokines, NF-KB pathway

Tropical Journal of Pharmaceutical Research is indexed by Science Citation Index (SciSearch), Scopus, International Pharmaceutical Abstract, Chemical Abstracts, Embase, Index Copernicus, EBSCO, African Index Medicus, JournalSeek, Journal Citation Reports/Science Edition, Directory of Open Access Journals (DOAJ), African Journal Online, Bioline International, Open-J-Gate and Pharmacy Abstracts

\section{INTRODUCTION}

Psidium guajava Linn., commonly known as guava, has been naturalized in South - East Asia
[1]. In oriental regions, such as Taiwan, guava leaves is boiled and the extract is used in folk medicine for the treatment of diabetes [2] but few of the extract has been investigated for their 
preclinical or clinical efficacy. Psidium guajava Linn. (Myrtaceae) is a small evergreen tree and its leaves are short-petiolate with the blade oval with prominent pinnate veins. Leaves of this plant have been reported to contain several compounds such as triterpenoids [1] and flavonoids [3]. Recent tropical relevance studies have shown that the leaves of Psidium guajava have been used for the treatment of a number of diseases, e.g. as an anti - inflammatory, for diabetes, pain relief and anti - pyretic. Moreover, previous phytochemical investigations had demonstrated that triterpenoids are the principle compounds isolated from the leaves [4]. As a continuing research of this potential drug for diabetic complications, we conduct this study on 5 known triterpenes: Asiatic acid, Maslinic acid, Corosolic acid, Oleanolic acid, and Ursolic acid.

Many of the pathophysiological pathways of diabetic neuropathy focused on inflammation and its mechanisms appear to converge upon the activation of NF - KB in diabetic neuropathy $[5,6]$. In addition to modulating of pro - inflammatory responses, NF - $\mathrm{kB}$ causes the transcription of pro - inflammatory mediators such as interleukin - 6 (IL - 6), tumor necrosis factor - a (TNF - a) and inducible nitric oxide synthase (iNOS) [7]. It is reported that Asiatic acid and Oleanolic acid possess anti - inflammatory activities [8,9]. Therefore, it is essential to investigate whether TTPGL has protective effect on DPN by suppressing NF - $\mathrm{kB}$ pathway and thus inhibits pro - inflammatory mediators.

\section{EXPERIMENTAL}

\section{Chemicals and reagents}

Protein kinase $B(A k t)$, inhibitory $k B$ kinase alpha (IKKa) and phosphor - IKKa and NF - KB - p65, horse radish per - oxidase (HRP) - conjugated antirabbit IgG were purchased from Cell Signaling Technology, Inc. (Beverly, MA, USA). Other reagents were purchased from Sigma Aldrich (St. Louis, MO, USA) unless indicated elsewhere.

\section{Extraction and isolation compounds of TTPGL}

The leaves of Psidium guajava were collected from their natural habitat in Guangzhou, Guangdong, in October of 2007. The plant was authenticated by Prof. Shu - Yuan Li (Guangdong Pharmaceutical University, Guangzhou, China). A voucher specimen (no. 2007101801) was deposited at the herbarium of Institute of Traditional Chinese Medicine \& Natural Products, Jinan University.
The air-dried leaves of $P$. guajava $(5.0 \mathrm{~kg})$ were powdered and percolated with $85 \%$ (v/v) EtOH at room temperature for three times $(3 \times 35$ liters). After removing the solvent, the alcohol extract $(110 \mathrm{~g})$ was suspended in $\mathrm{H}_{2} \mathrm{O}$ and partitioned successively by petroleum acetone (PA) to obtain the PA extract (88 g) and water extract (22 g), respectively. The PA extract (88 g) was separated by silica gel (200 - 300 mesh) CC and eluted with TFA - MeOH to obtain fractions. Fractions were separated by silica gel column with gradient mixtures of $\mathrm{MeOH}$ - TFA as eluents to yield subfractions. The PA extract $(88.0 \mathrm{~g})$ was separated by silica gel (200 - 300 mesh) CC and eluted with $\mathrm{MeOH}$ - TFA to obtain 8 fractions (C1 - C8). Fractions were subjected to silica gel column eluted with mixtures of $\mathrm{MeOH}$ - TFA (8500:1; v/v) to yield compounds 1 (120 mg, 98 $\%), 2$ (120 mg, $98 \%), 3$ (120 mg, $98 \%), 4$ (150 $\mathrm{mg}, 98 \%)$ and 5 (200 mg, $98 \%$ ).

\section{Induction of hyperglycemia in rats and blood glucose measurement}

Adult male Sprague Dawley rats (130 - $140 \mathrm{~g}$, animal quality certificate number SCXK 2013 0002) were maintained under standard condition (a constant temperature on a clean environment in a $12 \mathrm{~h}$ light/12 $\mathrm{h}$ dark cycle). All procedures were carried out in accordance with the principles of U.S. National Institutes of Health guidelines [10]. All study protocols were approved by the local government committee for animal research (approval ref no. 20140110103118). The rats were fasted for $12 \mathrm{~h}$ prior to injection, rats were injected with STZ (40 $\mathrm{mg} / \mathrm{kg}$, dissolved in $0.1 \mathrm{mmol} / \mathrm{L}$ sodium citrate buffer, $\mathrm{pH}$ 4.5) intraperitoneally, while the control group rats were injected with the vehicle citrate buffer. One week later, the rats with blood glucose above $11.1 \mathrm{mmol} / \mathrm{L}$ were considered diabetic and selected for further pharmacological studies [11]; secondly, animals were fed their respective diets until the end of the study as described: diabetic rats were fed with a high - fat diet (consisting of $70 \%$ standard laboratory diet, $15 \%$ carbohydrate, $10 \%$ lard and $5 \%$ yolk powder) while the healthy rats were fed with normal chow. Blood samples obtained from the tail vein of rats were monitored for glucose once a week using reagent strips with a glucometer (Onetouch Ultra 2, Johnson \& Johnson, Milpitas, CA, USA).

\section{Experimental animal groups}

Diabetic rats were randomized into 5 groups of 10 animals each and were still fed with a high fat diet, namely DM (untreated diabetic rats) group, TTPGL - 30 (30 mg/kg/d, i.g.) group, 
TTPGL - 60 (60 mg/kg/d, i.g.) group, TTPGL $120(120 \mathrm{mg} / \mathrm{kg} / \mathrm{d}$, i.g.) group and RSG (3 $\mathrm{mg} / \mathrm{kg} / \mathrm{d}$, i.g.) group (as a hypoglycemic positive control). Normal rats served as control group $(n=$ 10). 8 weeks were designed for each group. The samples from the rats were collected during anesthesia and finally the rats were sacrificed.

\section{Nerve function measurement}

We measured MNCV and SNCV in all rats at 1, 4 and 8 weeks after treatment in rats. MNCV was calculated by subtracting the distal latency from the proximal latency, and recorded in meters per second as the ratio of the distance in millimeters. SNCV was measured in the digital nerve to the second toe, using the smallest intensity current that resulted in an amplitude response, and was recorded in meters per second as the ratio of distance between stimulating and recording electrodes. [12,13]

To determine the thermal response latency, rats were placed within a plexi glass chamber and allowed to acclimatize for at least $20 \mathrm{~min}$. A thermal stimulation meter from ChengDu Technology \& Market Co., LTD. (Chengdu, China) was used. The device was activated after placing the stimulator directly beneath the tail. Tail flick latency in response to the radiant heat (a heating rate of $1.3^{\circ} \mathrm{C}$ per second, cut - off time of $16 \mathrm{~s}$ for rats) was recorded. At least three readings per rat were taken at $15 \mathrm{~min}$ intervals, and the average was calculated.

\section{Immunofluorescence (IF) staining}

We fixed and embedded sciatic nerves in paraffin with transverse section. The paraffin sections, cut at $5 \mu \mathrm{m}$, were deparaffinized in xylene and rehydrated in a series of graded alcohols to distilled water. Antigen retrieval was then performed in $10 \mathrm{mmol} / \mathrm{L}$ citrate, $\mathrm{pH} 6.0$, using 1100 - W microwave oven for $3 \mathrm{~min}$ at high power and $12 \mathrm{~min}$ at $50 \%$ power. The slides were allowed to cool at room temperature. Tissues were then incubated for $10 \mathrm{~min}$ in $0.3 \%$ hydrogen peroxide to block endogenous peroxidase activity. The slices were then washed for $5 \mathrm{~min}$ in PBS and were incubated in $0.1 \%$ Triton $X-100$ and $5 \%$ goat serum for $30 \mathrm{~min}$. Tissues were then incubated with primary monoclonal antibody raised against IL - 6 (1:100), TNF (1:100), iNOS (1:50) overnight at 4 ${ }^{\circ} \mathrm{C}$ in a humidified chamber. Following extensive washing, the slices were incubated with fluorescein conjugated secondary antibody at room temperature for $1.5 \mathrm{~h}$ [14]. The nuclei were counterstained using 4' - 6'diamidino - 2 phenylindole (DAPI). All immunofluorescence staining were performed in replicates on 3 sciatic nerves. The sections were examined under a Zeiss Axiophot fluorescence microscope. To examine inflammatory cytokines infiltration in the sciatic nerve, randomly 15 - 20 fields or focuses from each animal were observed and analyzed by Image $\mathrm{J}$ (version $1.46 \mathrm{~m}$ ) software.

\section{Western blotting assay}

Protein lysates were obtained by homogenizing sciatic nerves with lysis buffer containing $1 \%$ Triton X - 100, $150 \mathrm{mmol} / \mathrm{L} \mathrm{NaCl}, 1 \mathrm{mmol} / \mathrm{L}$ EDTA, $2.5 \mathrm{mmol} / \mathrm{L}$ sodium pyrophosphate, 1 $\mathrm{mmol} / \mathrm{L} \beta$ - glycerophosphate, $1 \% \mathrm{Na}_{3} \mathrm{VO}_{4}, 1$ $\mu \mathrm{g} / \mathrm{mL}$ leupeptin, $1 \mu \mathrm{g} / \mathrm{mL}$ aprotinin and 20 $\mathrm{mmol} / \mathrm{L}$ Tris $(\mathrm{pH} 7.5)$. Equal amounts of $40 \mu \mathrm{g}$ proteins were separated by SDS - PAGE (10\%) and transferred to a nitrocellulose membrane (Pall Life Sciences, Florida, USA). After blocking with $3 \%$ bovine serum albumin, membranes were incubated with primary antibodies against Akt, IKKa, NF - kB - p65, phosphor - Akt, phosphor - IKKa, and phosphor - NF - KB - p65 for $12 \mathrm{~h}$ at $4{ }^{\circ} \mathrm{C}$. After washing, membranes were then incubated with anti - rabbit IgG conjugated to HRP (1:1000 dilution) at room temperature for $1 \mathrm{~h}$. Membranes were washed thrice, visualized with Immobil on Western HRP Substrate, and scanned with a Kodak Digital Imaging System (Gel Logic 2200 Pro; Kodak, Tokyo, Japan). $\beta$ actin expression was set as internal control and the relative band densities were quantified by densitometry.

\section{Statistical analysis}

Statistical analysis was performed using SPSS 19.0 software (SPSS, Chicago, USA). Data are expressed as mean \pm SEM. Multiple groups were compared using one - way analysis of variance followed by Tukey HSD post hoc comparison. $P$ $<0.05$ was considered statistically significant.

\section{RESULTS}

The structures of compound $1-5$ were identified by comparing the ${ }^{1} \mathrm{H}$ NMR and ${ }^{13} \mathrm{C}$ NMR spectral data with previous literature reports. Principal components are shown in Table 1.

\section{Effect of TTPGL on fasting blood glucose (FBG)}

Levels of FBG in serum are shown in Fig 1. It showed significant differences $(p<0.05)$ in the TTPGL - 60, TTPGL - 120 and RSG groups compared to the DM group. 
Table 1: Characterization of principal components in TTPGL by HPLC

\begin{tabular}{llccc}
\hline No. & Chemical & $\begin{array}{c}\text { Molecular } \\
\text { formula }\end{array}$ & $\begin{array}{c}\text { Molecular } \\
\text { weight }\end{array}$ & Content (\%) \\
\hline $\mathbf{1}$ & Asiatic acid & $\mathrm{C}_{30} \mathrm{H}_{48} \mathrm{O}_{5}$ & 488 & 18 \\
$\mathbf{2}$ & Maslinic acid & $\mathrm{C}_{30} \mathrm{H}_{48} \mathrm{O}_{4}$ & 472 & 12 \\
$\mathbf{3}$ & Corosolic acid & $\mathrm{C}_{30} \mathrm{H}_{48} \mathrm{O}_{4}$ & 472 & 36 \\
$\mathbf{4}$ & Oleanolic acid & $\mathrm{C}_{30} \mathrm{H}_{48} \mathrm{O}_{3}$ & 456 & 3 \\
$\mathbf{5}$ & Ursolic acid & $\mathrm{C}_{30} \mathrm{H}_{48} \mathrm{O}_{3}$ & 456 & 8 \\
\hline
\end{tabular}

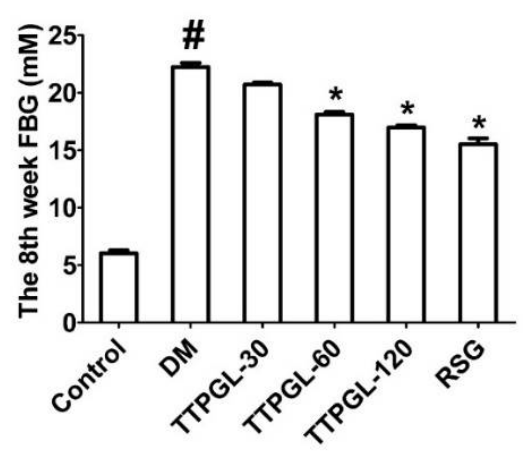

Fig 1: Effect of TTPGL on FBG. ${ }^{*} p<0.05$ vs. DM. . $p$ $<0.05$ vs. Control. 8 rats in each group. $\mathrm{mM}=\mathrm{mmol} / \mathrm{L}$

\section{Effect of TTPGL on neurological function}

The definition of DPN may include a focused neurological examination or nerve conduction studies, or, all of these [5]. As shown in Fig. 2, within 8 weeks of the onset of diabetes induced by STZ, a severe peripheral neuropathy developed. Rats with 8 - week duration of high fat/STZ diabetes had development of MNCV (16 $\%)$ and SNCV (9\%) deficits compared with
Control. In contrast, after 8 weeks of TTPGL 120/RSG treatment, all nerve conduction velocities demonstrated a marked improvement $(p<0.05)$ (Fig 2A and B). In addition, compared to DM group, treatment of TTPGL - 120 and RSG groups markedly $(p<0.05)$ produced a reduction in thermal latency (Fig. 2C).

\section{Effect of TTPGL on sciatic nerve TNF - $\alpha$, IL - 6 and iNOS expression}

Based on findings above, using IF staining, we confirmed the inhibitory effect of TTPGL on inflammatory mediators. Compared to the Control, DM group exhibited significant $(p<0.05)$ increase in fluorescence intensity, while treatment with TTPGL decreased the fluorescence intensity. Those micrographs indicated that TTPGL inhibited the production of the inflammatory mediators TNF - $\alpha, \mathrm{IL}-6$ and iNOS at the dose of 60 or $120 \mathrm{mg} / \mathrm{kg}$, respectively.

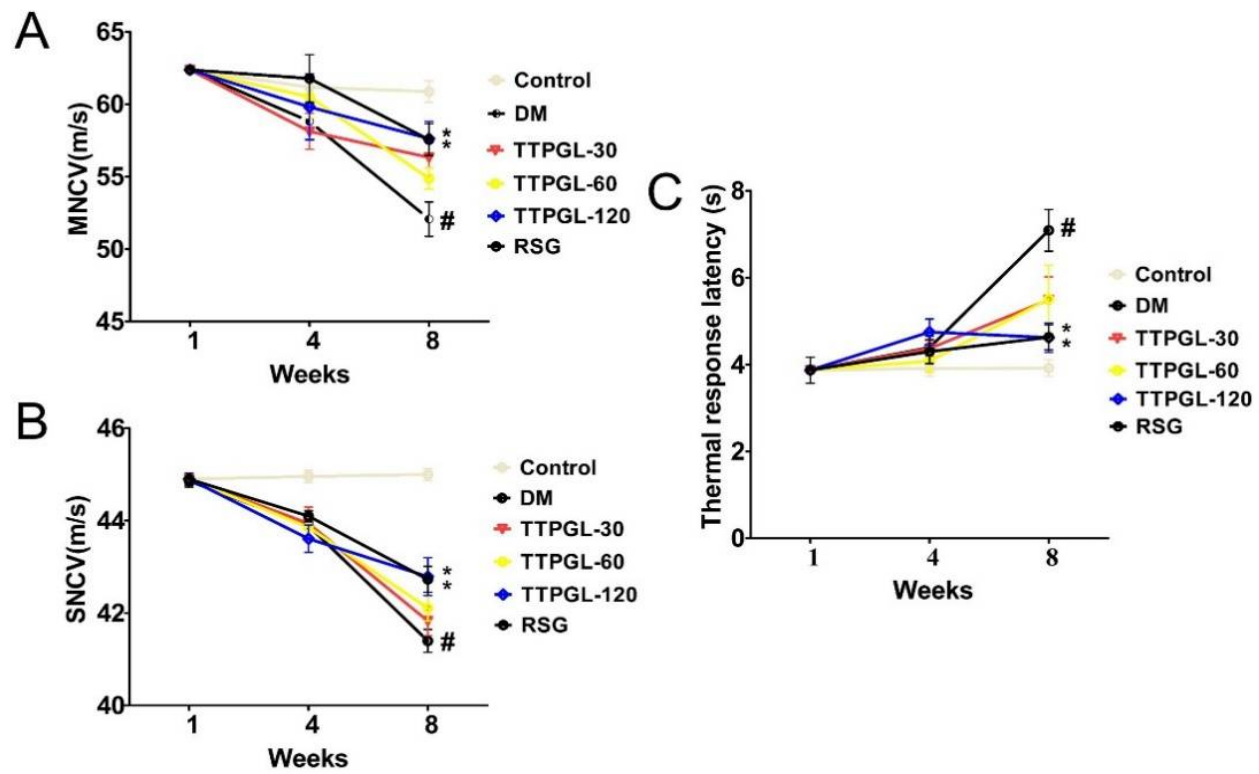

Fig 2: Effect of TTPGL on neurological functions. Nerve function test was performed at the $1^{\text {st }}, 4^{\text {th }}, 8^{\text {th }}$ week. Variables of peripheral nerve function in nondiabetic and diabetic rats maintained with or without TTPGL or RSG treatments. MNCV (A), SNCV (B) and Thermal response latency test (C). ${ }^{*} p<0.05$ vs. DM. ${ }^{*} p<0.05$ vs. Control. 8 rats in each group 
Quantitative analysis of inflammatory expressions are shown in Fig. 3. RSG effects on high - fat diet/STZ induced diabetic rats is similar to that of TTPGL (60 or $120 \mathrm{mg} / \mathrm{kg}$ ). These data are in accordance to our previous hypothesis that TTPGL could be developed into a protective agent against NF - $\mathrm{KB}$ - mediated expression of neuroinflammatory mediators.

\section{Effect of TTPGL on NF - KB activation via the Akt/IKKa pathway}

As shown in Fig. 4, the experiments revealed that the constitutive NF - $\mathrm{KB}$ cascade in sciatic nerve of diabetic rats was significantly $(p<0.05)$ increased when compared with that of the
Control. TTPGL dose - dependently decreased $(p<0.05$ or $p<0.01)$ the phosphorylation levels of Akt (Fig. 4A), IKKa (Fig. 4B) and which subsequently suppressed phosphorylation levels of NF - $\mathrm{kB}$ - p65 (Fig. 4C). RSG as well as TTPGL $(60 \mathrm{mg} / \mathrm{kg}, 120 \mathrm{mg} / \mathrm{kg})$ significantly $(p<$ 0.05 ) inhibited the phosphorylation levels of Akt, IKKa and NF - KB - p65 than that of the DM group (Fig. 4). Furthermore, TTPGL at dose of $120 \mathrm{mg} / \mathrm{kg}$ exhibited extremely significant $(p<$ $0.01)$ decrease in protein expression. These findings suggested that TTPGL suppresses the $N F-K B$ - p65 activation and regulate NF - KB p65 activity through Akt//KKa pathway in a dosedependent manner.

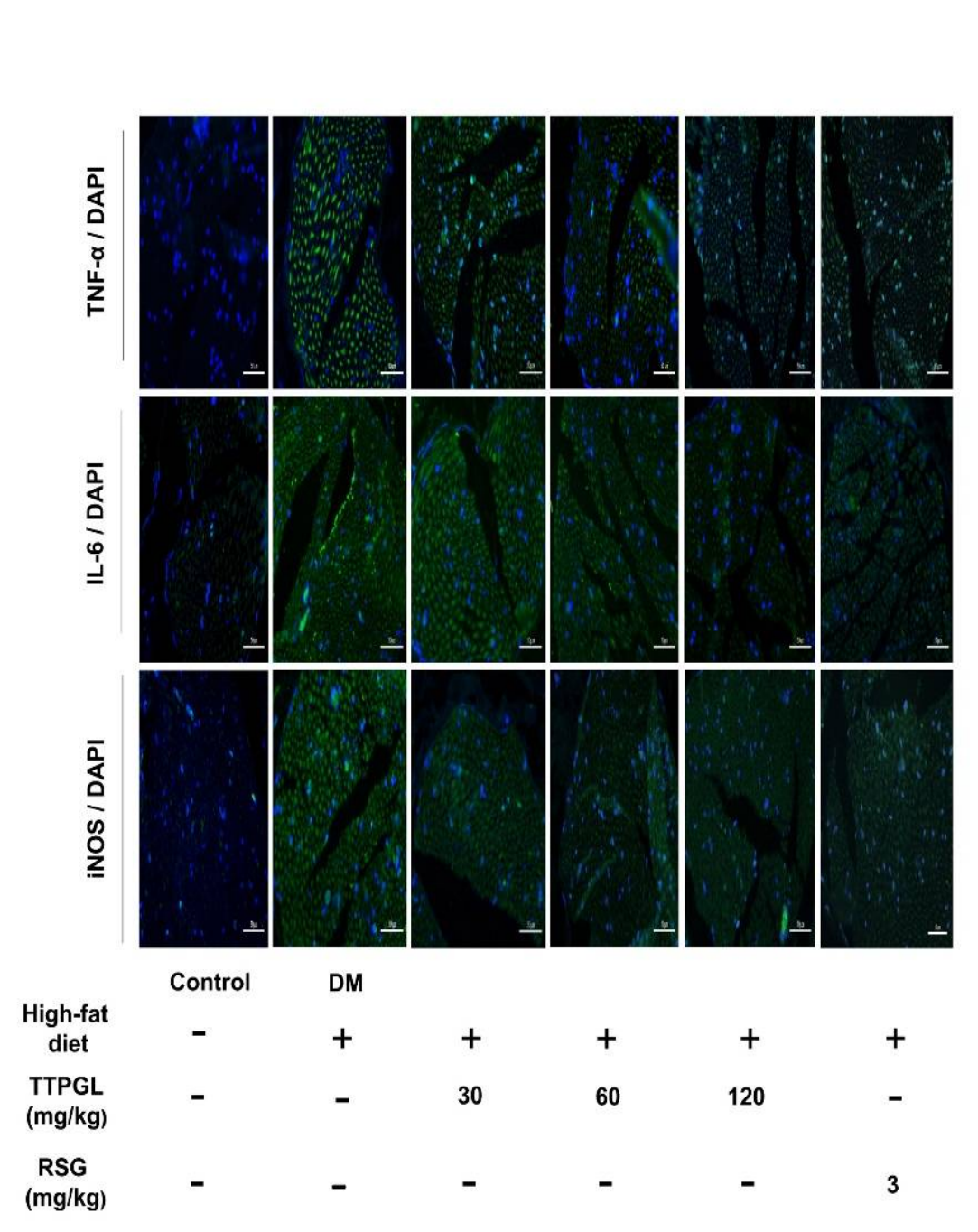

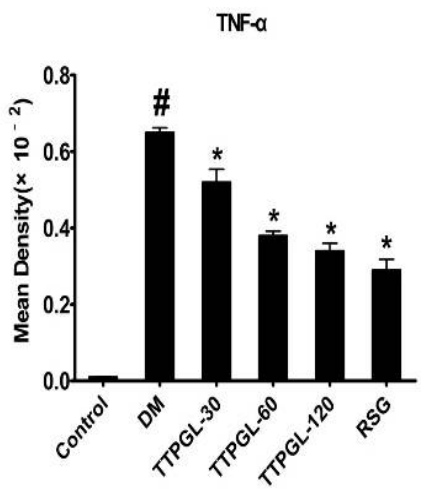

IL-6
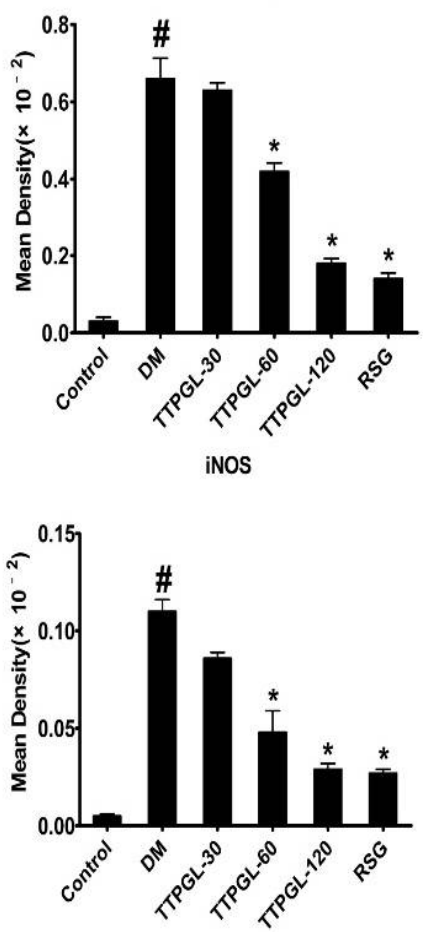

Fig. 3: Immunofluorescence evaluation of sciatic nerves. Transverse section was conducted in this study, Left: Immunofluorescent micrographs showing TNF - $\alpha$, iNOS and IL - 6 expressions in each group. The location of neuroinflammatory mediators (green) were examined by immunofluorescence staining and nuclei were stained with DAPI (blue). Bar, $50 \mu \mathrm{m}$. Right: Quantitative analysis of neuroinflammatory mediator expressions according to immunofluorescent micrographs, respectively. ${ }^{*} p<0.05$ vs. DM; ${ }^{\#} p<0.05$ vs. control 


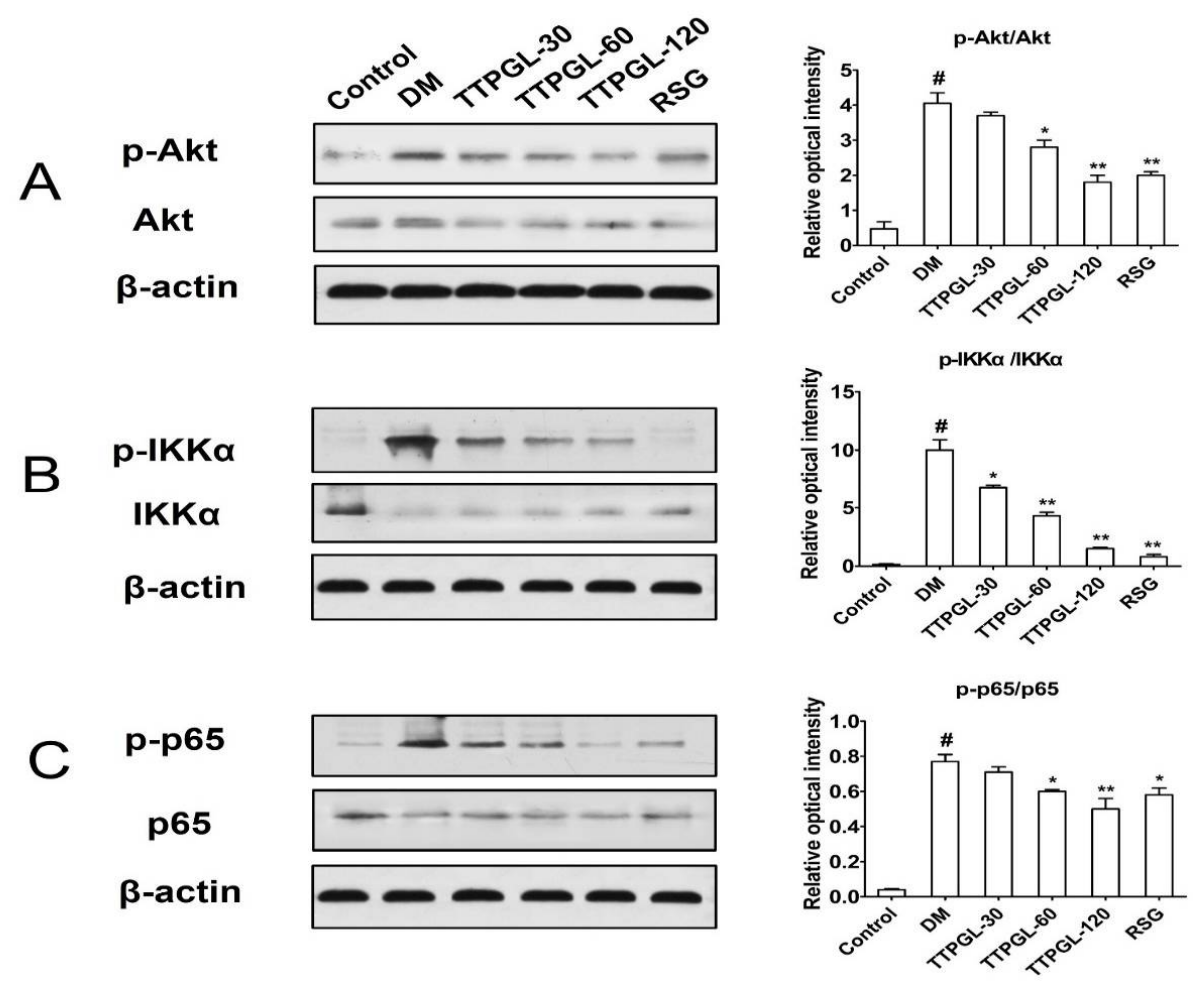

Fig. 4: Evaluation of protein expression by using western blot. (A) Left: The expression of $p$ - Akt and Akt; Right: Quantitative analysis of $p$ - Akt / Akt. (B) Left: The expression of $p$ - IKKa and IKKa; Right: Quantitative analysis of $p$ - IKKa / IKKa. (C) Left: The expression of $p$ - p65 and p65; Right: Quantitative analysis of p - p65 / p65. "* $p<$ 0.01 and ${ }^{*} p<0.05$ vs. DM; ${ }^{*} p<0.05$ vs. control

\section{DISCUSSION}

The findings reported herein show that long term TTPGL treatment exerted protective effects in high - fat/STZ relevant model for DPN in vivo. Through a combination of biochemical and histological results, TTPGL plays a role in suppressing the expression of inflammatory mediators. Mechanistic studies suggested that TTPGL significantly ameliorates DPN through negatively regulating $A k t / I K K a$ mediated $N F-K B$ pathway.

In present studies, treatment with TTPGL - 120 ameliorated the deficits in MNCV, SNCV versus the DM group. The sensorimotor alteration, is one of the debilitating features of diabetic neuropathy in which nerve sensitization and inflammation are the commonly observed mechanisms $[15,16]$. The favorable effects of TTPGL on nerve - functional deficits may be due to its lightly hypoglycemic effect and its effect on inflammatory infiltration. The exact principle responsible for the hypoglycemia and amelioration effect on DPN were not investigated in the current study. Moreover, further studies would be required to determine the exact mechanism(s) and site of action of the extract.
Inflammatory cytokines which have been observed to contribute to the development of neuropathy and neuropathic pain in diabetes includes TNF - $\alpha$ and IL - 6 [17]. Treatment with TTPGL was able to attenuate the increased levels of TNF - $\alpha$ and IL -6 in treated group which might have aided in decreasing inflammatory damage found in sciatic nerves. It is known that NF - $\mathrm{kB}$ activation induces the expression of iNOS, an enzyme induced during inflammatory conditions which specifically binds to COX-2 and S - nitrosylates to enhance COX 2 activity [18]. TTPGL - 120 treatment inhibited the induction of iNOS which may have further decreased COX - 2 activity as well as inflammatory damage in neurons.

In this study, we observed TTPGL ameliorative effect and investigated correlated underlying mechanisms in the diabetes peripheral neuropathy rats. The NF - KB signaling cascade interacts with several parallel pathways including the signaling cascades initiated by phosphatidylinositol 3 - kinase PI3K and Akt $[19,20]$. Akt, functioning as a key downstream target of $\mathrm{PI} 3 \mathrm{~K}$, has been suggested to function as an IKK (IKB kinase) kinase [21]. Western blot analysis showed that the expressions of $p$ - Akt and $p-I K K \alpha$ in the DM group were significantly higher $(p<0.01)$ than those in the Control. In 
addition, the increased expressions of $p$ - Akt and $\mathrm{p}$ - IKKa after STZ treatment could be attenuated by TTPGL administration. These results were indicated that TTPGL exerts ameliorative effect in DPN rats associated with changes in NF - $\mathrm{KB}$ cascade.

\section{CONCLUSION}

The findings provide the first direct evidence that TTPGL effectively decreased the release of inflammatory mediators in vivo. The efficacy of TTPGL, at least in part, may be due to the combined modulation of levels of NF - KB and inflammatory mediators as seen in our study. Interactions among TTPGL and other inflammatory mediators implicated in the pathogenesis of DPN also need to be explored. However, this work provides rationale for the evaluation of TTPGL in easily accessible natural sources as agents for diabetic complicates.

\section{ACKNOWLEDGEMENT}

Financial support of this research was provided by grants from National Natural Science Foundation of China (no. 81373935), Educational Commission of Guangdong Province (no. cxzd1111) and Program for New Century Excellent Talents in University (no. NCET - 12 0676). The authors would also like to thank Institute of Traditional Chinese Medicine \& Natural Products, Jinan University for supporting this research.

\section{REFERENCES}

1. Begum S, Hassan SI, Siddiqui BS, Shaheen F, Ghayur $M N$, Gilani AH. Triterpenoids from the leaves of Psidium guajava. Phytochemistry, 2002; 61: 399-403.

2. Shen S-C, Cheng F-C, Wu N-J. Effect of Guava (Psidium guajava Linn.) Leaf Soluble Solids on Glucose Metabolism in Type 2 Diabetic Rats. Phytother Res, 2008; 22: 1458-1464.

3. Lozoya X, Meckes M, Abou-Zaid M, Tortoriello J, Nozzolillo C, Arnason JT. Quercetin glycosides in Psidium guajava $L$. leaves and determination of a spasmolytic principle. Arch Med Res, 1994; 25: 11-15.

4. Gutierrez RMP, Mitchell S, Solis RV. Psidium guajava: $A$ review of its traditional uses, phytochemistry and pharmacology. J Ethnopharmacol, 2008; 117: 1-27.

5. Edwards JL, Vincent AM, Cheng HT, Feldman EL. Diabetic neuropathy: Mechanisms to management. Pharmacol Ther, 2008; 120: 1-34.

6. Cameron NE, Cotter MA. Pro-inflammatory mechanisms in diabetic neuropathy: Focus on the nuclear factor kappa B pathway. Curr Drug Targets, 2008; 9: 60-67.
7. Karin M, Yamamoto $Y$, Wang QM. The IKKNF-kappa $B$ system: A treasure trove for drug development. Nat Rev Drug Discov, 2004; 3: 17-26.

8. Tsao S-m, Yin M-c. Antioxidative and Antiinflammatory Activities of Asiatic Acid, Glycyrrhizic Acid, and Oleanolic Acid in Human Bronchial Epithelial Cells. J Agric Food Chem, 2015; 63: 3196-3204.

9. Ramachandran V, Saravanan R. Efficacy of asiatic acid, a pentacyclic triterpene on attenuating the key enzymes activities of carbohydrate metabolism in streptozotocininduced diabetic rats. Phytomedicine, 2013; 20: 230236.

10. Anjaneyulu M, Berent-Spillson A, Inoue $T$, Choi J, Cherian K, Russell JW. Transforming growth factor-beta induces cellular injury in experimental diabetic neuropathy. Exp Neurol, 2008; 211: 469-479.

11. Wu D, Wen W, Qi C-L, Zhao R-X, Lu J-H, Zhong C-Y, Chen $Y$-Y. Ameliorative effect of berberine on renal damage in rats with diabetes induced by high-fat diet and streptozotocin. Phytomedicine, 2012; 19: 712-718.

12. Boulton AJM, Vinik Al, Arezzo JC, Bril V, Feldman EL, Freeman R, Malik RA, Maser RE, Sosenko JM, Ziegler $D$. Diabetic neuropathies - A statement by the American Diabetes Association. Diabetes Care, 2005; 28: 956962.

13. Lupachyk S, Watcho $P$, Stavniichuk $R$, Shevalye $H$, Obrosova IG. Endoplasmic Reticulum Stress Plays a Key Role in the Pathogenesis of Diabetic Peripheral Neuropathy. Diabetes, 2013; 62: 944-952.

14. Xu Y, Lei H, Guan R, Gao Z, Li H, Wang L, Song W, Gao $B$, Xin Z. Studies on the mechanism of testicular dysfunction in the early stage of a streptozotocin induced diabetic rat model. Biochem Biophys Res Commun, 2014; 450: 87-92.

15. Vinik Al, Mehrabyan A. Diabetic neuropathies. Med Clin North Am, 2004; 88: 947-+.

16. Vincent $A M$, Feldman EL. New insights into the mechanisms of diabetic neuropathy. Rev Endocr Metab Disord, 2004; 5: 227-236.

17. Verri WA, Jr., Cunha TM, Parada CA, Poole S, Cunha FQ, Ferreira SH. Hypernociceptive role of cytokines and chemokines: Targets for analgesic drug development? Pharmacol Ther, 2006; 112: 116-138.

18. Kim SF, Huri DA, Snyder SH. Inducible nitric oxide synthase binds, S-nitrosylates, and activates cyclooxygenase-2. Science, 2005; 310: 1966-1970.

19. Kane $L P$, Shapiro VS, Stokoe $D$, Weiss A. Induction of NF-kappaB by the Akt/PKB kinase. Curr Biol, 1999; 9: 601-604.

20. Romashkova JA, Makarov SS. NF-kappaB is a target of $A K T$ in anti-apoptotic PDGF signalling. Nature, 1999; 401: 86-90.

21. Ozes ON, Mayo LD, Gustin JA, Pfeffer SR, Pfeffer LM, Donner DB. NF-kappaB activation by tumour necrosis factor requires the Akt serine-threonine kinase. Nature, 1999; 401: 82-85. 